\title{
Évolution des cancers ano-génitaux reliés à l'infection au VPH déclarés au Québec - Incidence et survie
}

R Louchini, M.Sc; P Goggin, M.D., M.Sc.; M Steben, M.D. (1)

\section{Résumé}

Les cancers ano-génitaux (anus, vulve, vagin, verge) associés au virus du papillome humain (VPH) autres que les cancers du col, pour lequel le VPH a été reconnu la cause nécessaire à la cancérogénèse, sont peu documentés en raison de leur incidence plutôt faible. Le but de cette étude est de décrire l'incidence de ces cancers, de 1984 à 2001 et la survie relative qui leur est associée de 1984 à 1998 au Québec (Canada). L'incidence de ces cancers est en augmentation pour le cancer anal chez les femmes et plus récemment (depuis 1993-95) pour le cancer de la vulve. La survie relative à 5 ans a diminué, entre la période 1984-86 et la période 1993-95, chez les hommes de 57 \% à $46 \%$ pour le cancer anal et de $75 \%$ à 59 \% pour le cancer de la verge. Par contre, pendant la même période chez les femmes, la survie relative à 5 ans s'est améliorée pour le cancer anal, passant ainsi de $56 \%$ à $65 \%$. Elle est restée stable pour les cancers du col utérin et de la vulve respectivement autour de $74 \%$ et $82 \%$.

\section{Mots clés : alphapapillomavirus, néoplasme utérine cervicale, néoplasme anal, néoplasme vaginal, néoplasme de la vulve, néoplasme de la verge, survie}

\section{Introduction}

Depuis que le virus du papillome humain (VPH) a été reconnu comme la cause nécessaire du cancer du col utérin, les efforts de lutte contre cette maladie ont pris un virage majeur. En effet, en plus d'inclure des tests de détection du VPH dans la stratégie de dépistage ou de suivi des anomalies cytologiques, ces efforts ont été bonifiés par l'arrivée de vaccins prophylactiques ${ }^{(1)}$.

Le VPH est associé à un spectre de maladies qui dépasse le cancer du col utérin. On le croit maintenant associé aux autres cancers ano-génitaux; à plus de $80 \%$ des cancers de l'anus et à environ 40 à $50 \%$ des cancers de la verge, de la vulve et du vagin ${ }^{(2,3)}$. Il est aussi associé à une proportion significative de cancers oropharyngés ${ }^{(2,3)}$ et pourrait être associé à d'autres sièges de cancer ${ }^{(4)}$.
Jusqu'à ce jour, les cancers ano-génitaux associés au VPH autres que les cancers du col sont peu documentés en raison de leur incidence plutôt faible. Quelques études ont rapporté un accroissement de l'incidence du cancer de l'anus au cours des dernières décennies aux États-Unis ${ }^{(5-7)}$, en Suède ${ }^{(8)}$ et au Danemark ${ }^{(9)}$, mais peu de données populationnelles sont disponibles pour les autres sièges de cancers anogénitaux (vulve, vagin, verge) ${ }^{(10-12)}$.

Le but de cette étude est de décrire l'importance et l'évolution des cancers ano-génitaux reliés au VPH au Québec. Plus spécifiquement, ce portrait épidémiologique portera sur l'incidence des cancers du col utérin, de l'anus, de la vulve, du vagin et de la verge au Québec de 1984 à 2001 et sur la survie relative associée à ces cancers pour la période de 1984 à 1998. L'évolution de l'incidence du cancer peut révéler des indices sur le changement des facteurs de risques en cause. Elle peut également contribuer à évaluer les effets des interventions de dépistage, de diagnostic et de la prévention de ces cancers.

\section{Méthode}

\section{Source des données}

Les données d'incidence des cancers diagnostiqués au Québec entre 1984 et 2001 inclusivement proviennent du Fichier des tumeurs du Québec (FiTQ). Les données de population, utilisées pour le calcul des taux, sont les estimés de l'Institut de la statistique du Québec (ISQ), basés sur les recensements canadiens (Statistique Canada) et corrigés pour tenir compte du sous-dénombrement, du sur-dénombrement et des résidents non permanents.

Pour le calcul de la survie, le statut vital d'une personne atteinte d'un cancer et ayant fait l'objet d'une déclaration au FiTQ entre 1984 et 1998 a été déterminé par le raccordement du dossier d'incidence avec le fichier des décès du Québec de 1984 à 1999 inclusivement. Le raccordement entre ces deux fichiers est décrit dans le rapport intitulé « La survie au cancer pour les nouveaux cas déclarés au Québec en $1992 »^{(13)}$ à la section sur la qualité des données utilisées. Les sièges qui font l'objet de cette étude sont le cancer anal (CIM-9 154.2, 154.3, et 154.8), le cancer du col utérin (CIM-9 180), le cancer du vagin (CIM-9 184.0), le cancer de la vulve (CIM-9 184.1, 184.2, 184.3 et 184.4) et le cancer de la verge (CIM-9 187.1, 187.2, 187.3 et $187.4,187.7$, et 187.8 ). 


\section{Calcul des taux d'incidence standardisés}

Pour le calcul des taux standardisés, la population de référence est celle du recensement de 1991, avec la répartition par groupe d'âge de la population du Canada, sexes réunis. Pour chaque siège de cancer, les taux d'incidence ont été calculés selon trois catégories de morphologies (de type épidermoïde, de type adénocarcinome et autres types). Les cancers de type épidermoïde sont les plus fréquents (67\% , $58 \%, 64 \%, 57 \%$ et $74 \%$ respectivement pour les cancers de la vulve, du vagin, du col, anal et de la verge).

\section{Calcul de la survie}

Le calcul de la survie porte sur les nouveaux cas de cancer diagnostiqués et déclarés au Fichier des tumeurs du Québec pour les personnes âgées de moins de 100 ans au moment du diagnostic. Seul le premier cancer primaire déclaré chez un individu entre 1984 et 1998 inclusivement est retenu. Les cancers primaires subséquents sont exclus.

La survie relative est un ratio de la probabilité de survie observée chez un groupe de patients et de la probabilité de survie attendue d'un groupe comparable de la population générale qui hypothétiquement n'est pas atteinte du cancer étudié et qui présente les mêmes caractéristiques démographiques que le groupe de patients atteints de cancer. La survie observée est calculée selon la méthode actuarielle, soit le rapport entre le nombre de survivants à la fin de l'intervalle de suivi et le nombre de personnes vivantes au début de ce même intervalle. La survie attendue a été estimée, en utilisant trois tables de mortalité de la population générale du Québec ${ }^{(14-16)}$ selon la méthode de Ederer $\mathrm{II}^{(17)}$. Le calcul de la survie relative a été décrit de façon détaillée par Louchini et coll. ${ }^{(18)}$.

\section{Résultats}

\section{Incidence et survie relative des cancers ano-génitaux}

De 1984 à 2001, on dénombre 9556 cas de cancer primaire ano-génital, tous types confondus, dont 988 (10\%) chez les hommes et 8568 (90\%) chez les femmes. Cette forte proportion chez les femmes est attribuable principalement aux cas de cancer du col utérin qui à lui seul représente $65 \%$ de l'ensemble des cancers ano-génitaux. Le tableau 1 présente la répartition de ces cancers selon le siège, le sexe et la période, et la figure 1 les répartitions selon le siège, le sexe et l'âge pour toute la période. À l'exception du cancer du col utérin qui est plus fréquent chez les femmes de 40 à 59 ans, les cancers ano-génitaux sont plus fréquents chez les 60 à 79 ans où on trouve environ la moitié de l'ensemble de ces cancers.

En termes de taux d'incidence, le cancer du col vient en tête des cancers anogénitaux, suivi du cancer de la vulve. Par exemple, pour la période de 1999 à 2001, le taux d'incidence est de 5,6 par 100000 personnes pour le cancer du col utérin et de 1,3 par 100000 pour le cancer de la vulve. Les taux d'incidence des autres cancers varient entre 0,2 par 100000 et 0,8 par 100000 (figure 2).

Un total de 7201 cas de cancers anogénitaux, déclarés comme premier cancer primaire, ont été utilisés pour le calcul de la survie relative durant la période de 1984 à 1998. Ils sont répartis comme suit : 4822 cas de cancer du col utérin, 900 cas cancer de la vulve, 290 cas de cancer du vagin, 360 cas de cancer de la verge et 759 de cancer de l'anus (338 hommes et 421 femmes). La survie relative varie selon le siège et le sexe. La survie relative à 5 ans est plus élevée pour le cancer de la vulve (81\%) suivi du col utérin (72\%), de la verge (67\%), du cancer anal (53\% chez les hommes et $60 \%$ chez les femmes), et enfin du vagin (49\%). La figure 3 présente pour chaque siège de cancer anogénital la survie relative jusqu'à 15 ans

[suite, p. 116]

TABLEAU 1

Répartition du nombre de nouveaux cas (\% entre parenthèses) de cancer selon le siège (tous types de morphologies), le sexe et la période, Québec, 1984-1986 à 1999-2001

\begin{tabular}{|c|c|c|c|c|c|c|c|c|c|c|c|c|c|c|c|}
\hline \multirow{2}{*}{$\begin{array}{l}\text { Siège } \\
\text { Anal }\end{array}$} & \multirow{2}{*}{$\begin{array}{c}\text { Sexe } \\
\text { Hommes }\end{array}$} & \multicolumn{2}{|c|}{ 1984-86 } & \multicolumn{2}{|c|}{ 1987-89 } & \multicolumn{2}{|c|}{$1990-92$} & \multicolumn{2}{|c|}{ 1993-95 } & \multicolumn{2}{|c|}{ 1996-98 } & \multicolumn{2}{|c|}{ 1999-01 } & \multicolumn{2}{|c|}{ Total } \\
\hline & & 53 & (3) & 59 & (4) & 80 & (5) & 107 & (7) & 79 & (5) & 82 & (5) & 460 & (5) \\
\hline & Femmes & 70 & (4) & 69 & (5) & 101 & (7) & 111 & (7) & 101 & (7) & 139 & (9) & 591 & (6) \\
\hline Vagin & Femmes & 103 & (6) & 95 & (6) & 86 & (6) & 79 & (5) & 67 & (4) & 57 & (4) & 487 & (5) \\
\hline Vulve & Femmes & 182 & (10) & 189 & (12) & 181 & (12) & 196 & (12) & 264 & (17) & 263 & (16) & 1275 & (13) \\
\hline \multirow{2}{*}{ Total } & Femmes & 1584 & (92) & 1410 & (91) & 1371 & (89) & 1397 & (88) & 1371 & (89) & 1435 & (88) & 8568 & (90) \\
\hline & Total & 1714 & (100) & 1548 & (100) & 1544 & (100) & 1582 & (100) & 1543 & (100) & 1625 & (100) & 9556 & (100) \\
\hline
\end{tabular}


FIGURE 1

Répartition des nouveaux cas de cancer selon le siège (tous types de morphologies confondus), le sexe et l'âge, Québec, 1984-2001

a) Cancer anal chez les hommes

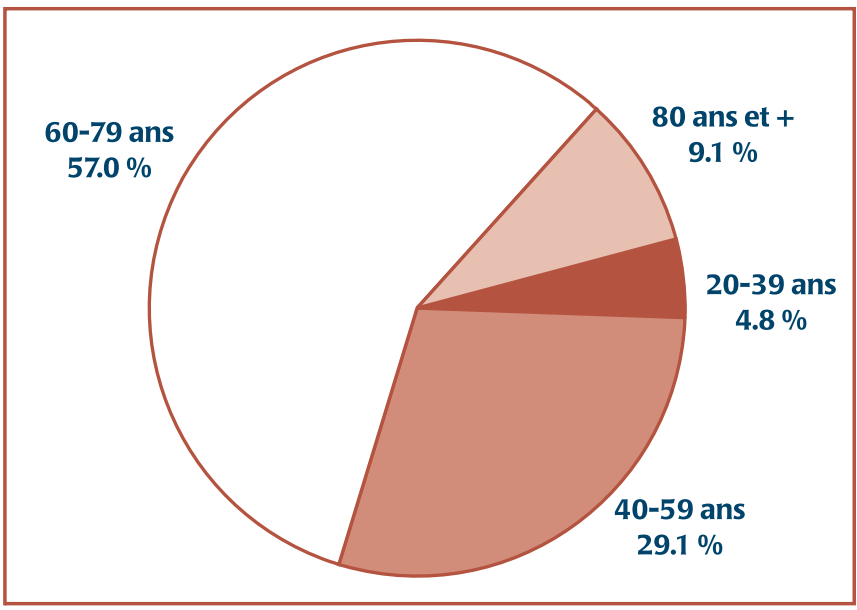

c) Cancer du col

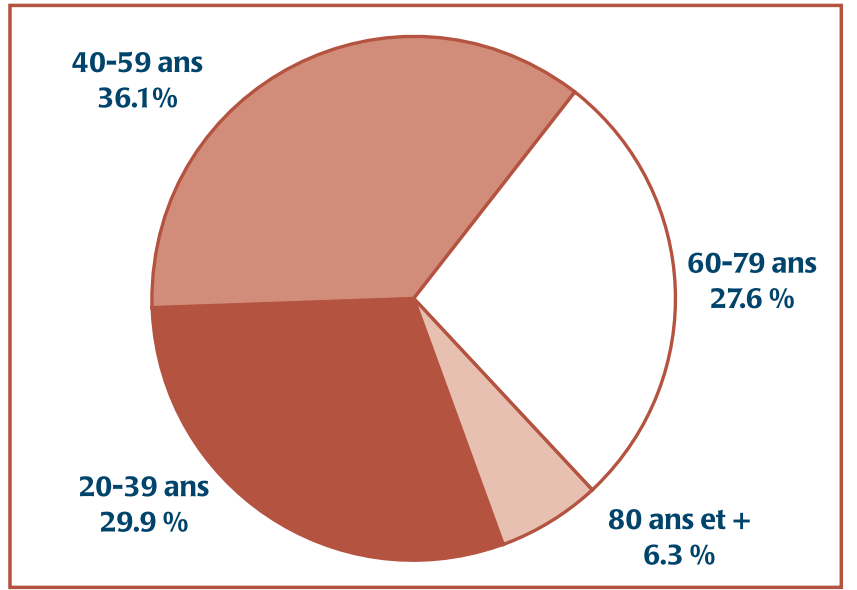

\section{e) Cancer de la vulve}

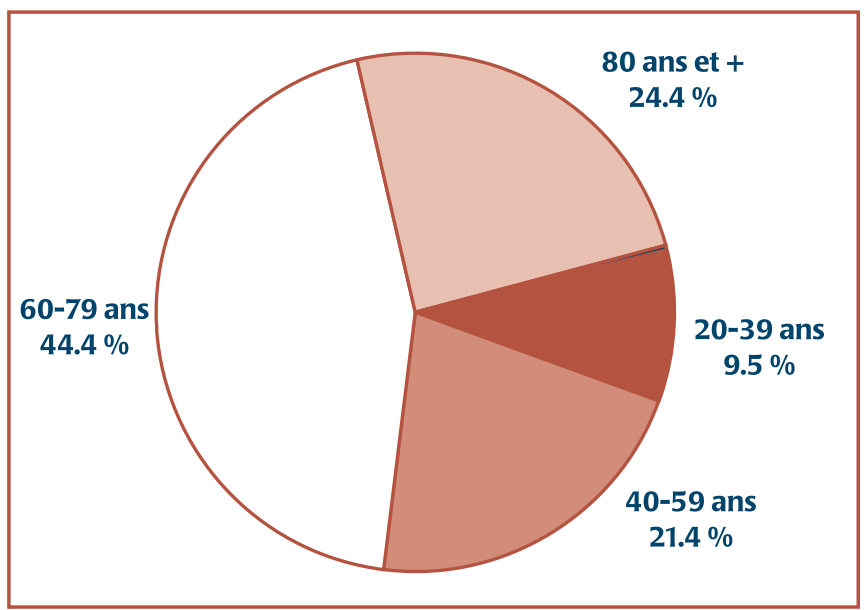

b) Cancer anal chez les femmes

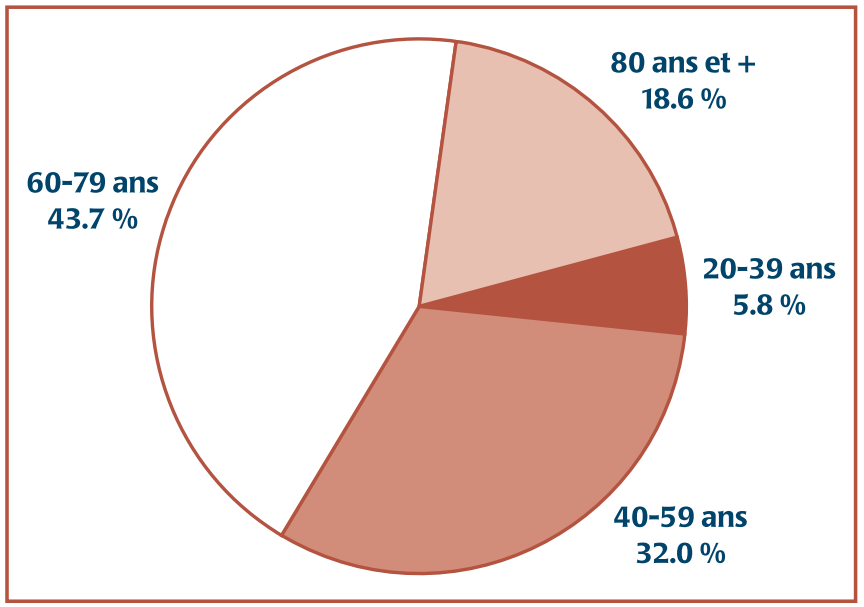

d) Cancer du vagin

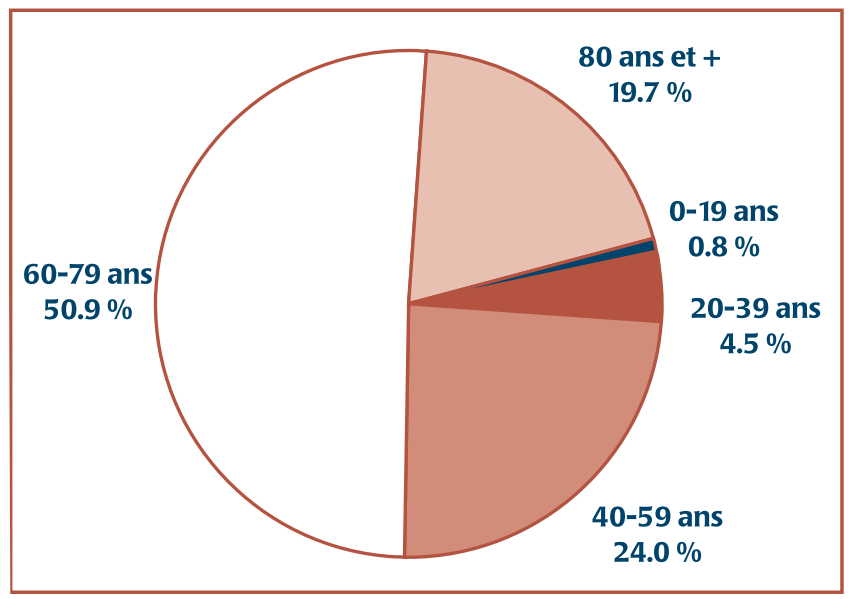

\section{f) Cancer de la verge}

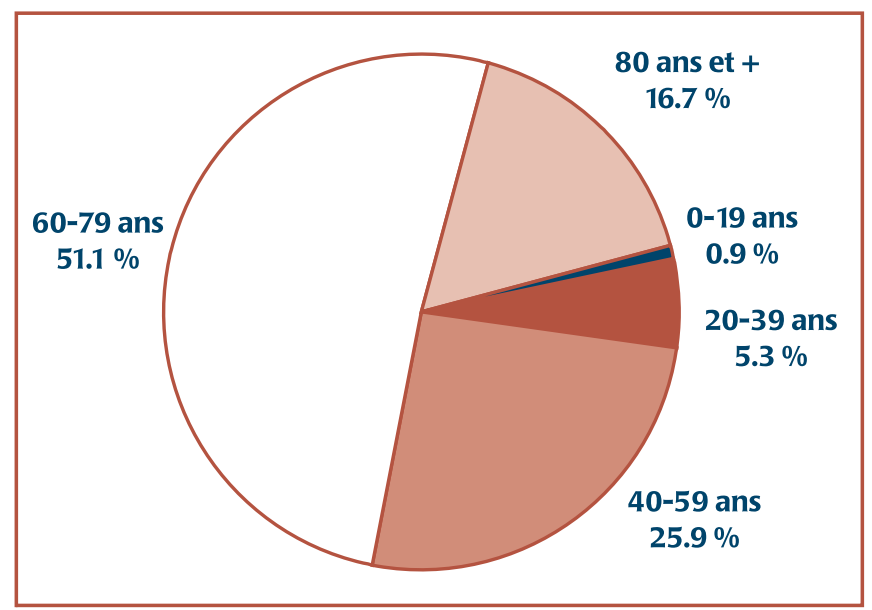


FIGURE 2

L'évolution des taux standardisés d'incidence des cancers ano-génitaux selon le sexe, Québec, de 1984-1986 à 1999-2001

a) Cancer anal - Hommes

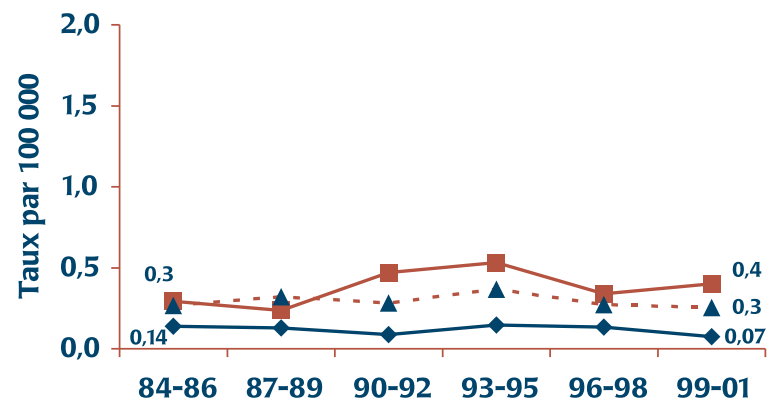

Année

$\longrightarrow$ Épidermoïde Adenocarcinomes - - A- - Autres

c) Cancer du col

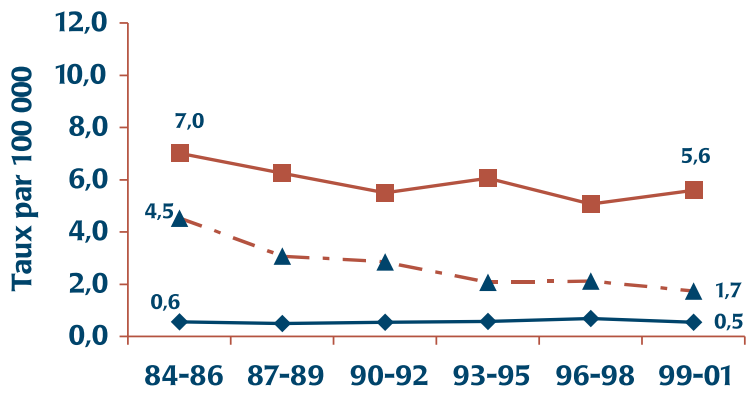

Année

$\longrightarrow$ Épidermoïde $\longrightarrow$ Adenocarcinomes $-\Delta-$ - Autres

e) Cancer de la vulve

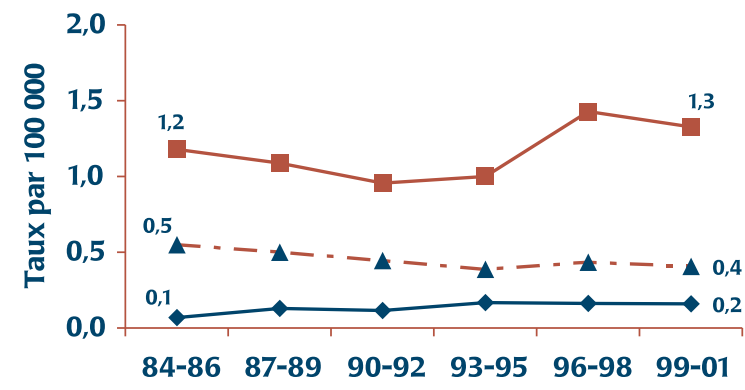

Année

$\longrightarrow$ Épidermoïde $\longrightarrow$ Adenocarcinomes $-\Delta--$ Autres b) Cancer anal - Femmes

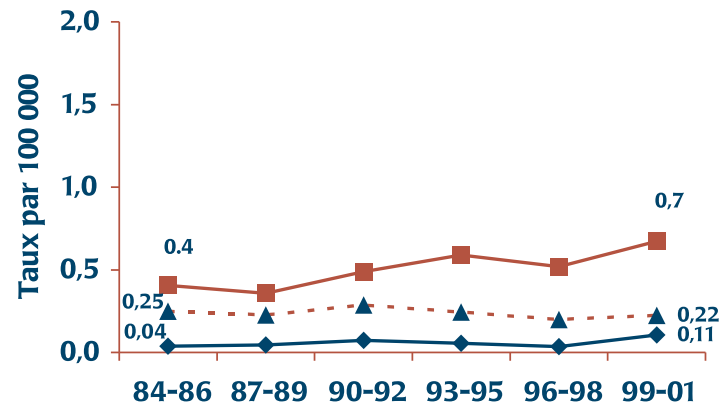

Année

$\longrightarrow$ Épidermoïde $\longrightarrow$ Adenocarcinomes - - $\mathbf{A}^{-}-$Autres

d) Cancer du vagin

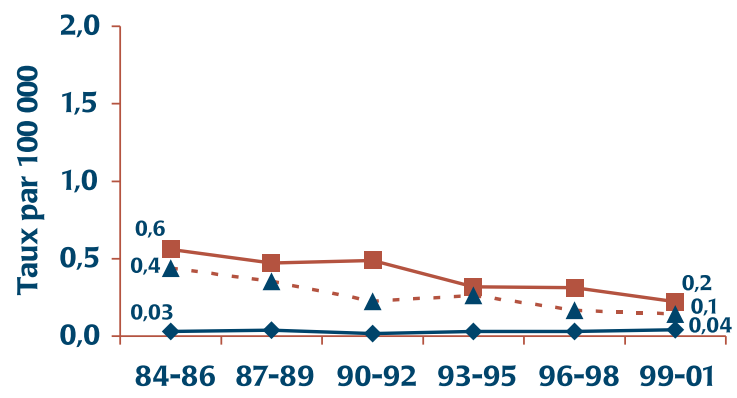

Année

$\longrightarrow$ Épidermoïde $\longrightarrow$ Adenocarcinomes - - $\mathbf{\Delta}-$ - Autres

f) Cancer de la verge

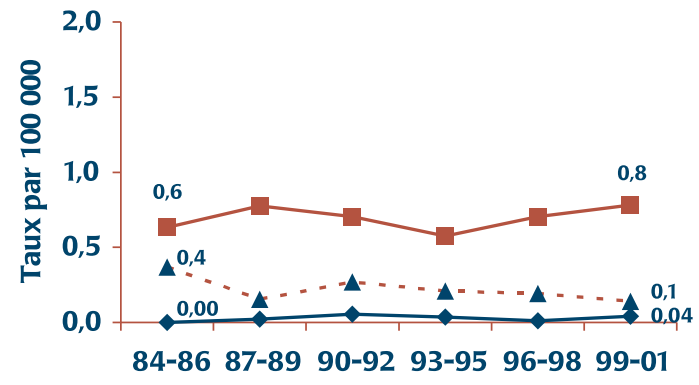

Année
$\square \longrightarrow$ Épidermoïde $\longrightarrow$ Adenocarcinomes $-\Delta^{--}$- Autres 
FIGURE 3

Survie relative (\%) selon le siège (tous types de morphologies) et le sexe, Québec, 1984-1998

a) Cancer anal chez hommes

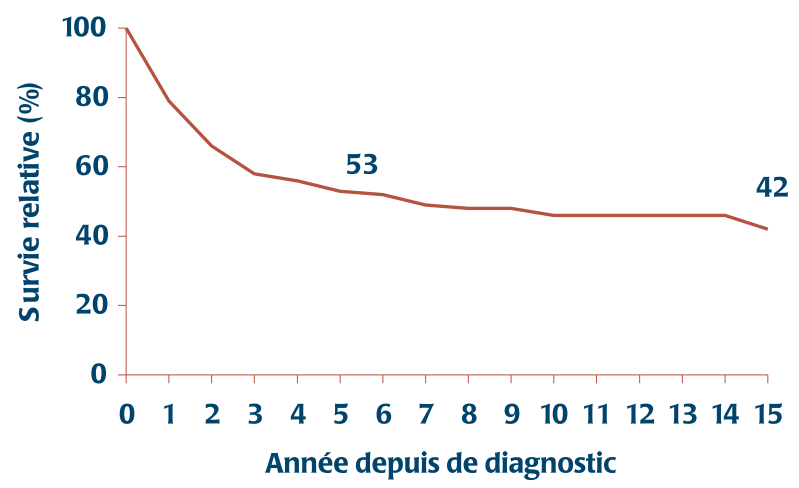

c) Cancer du col

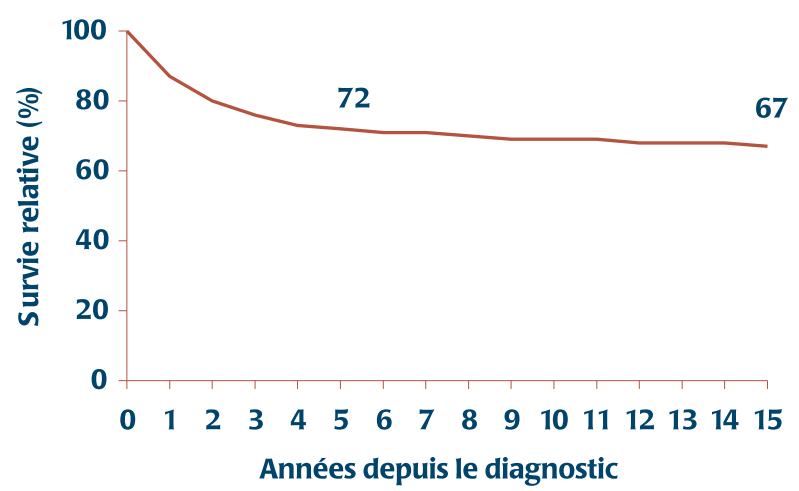

e) Cancer de la vulve

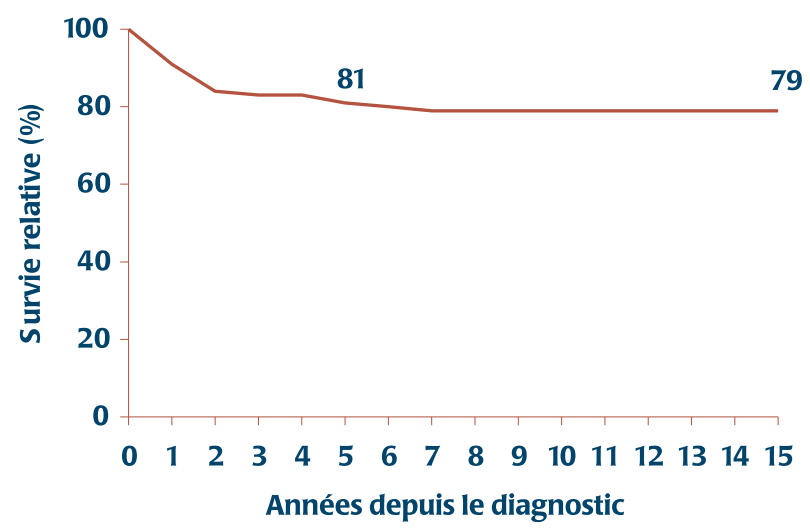

b) Cancer anal chez les femmes

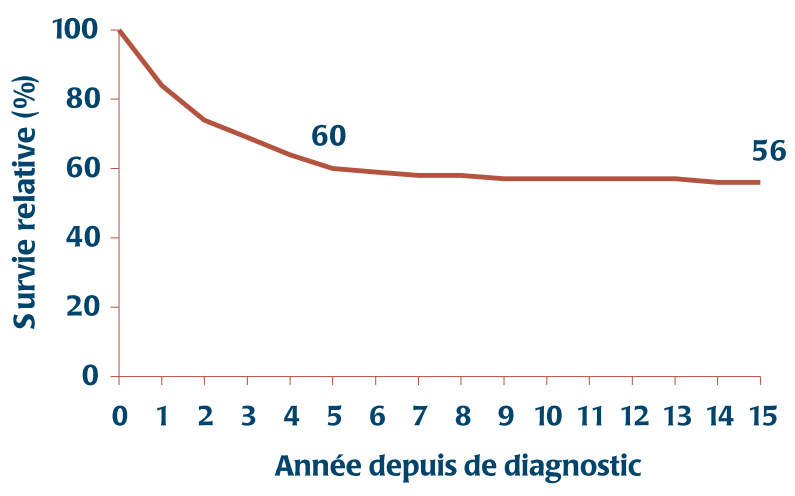

d) Cancer du vagin

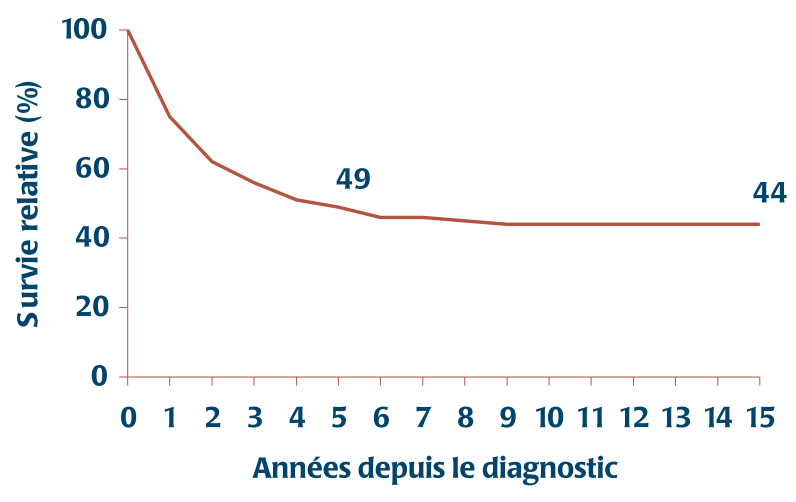

f) Cancer de la verge

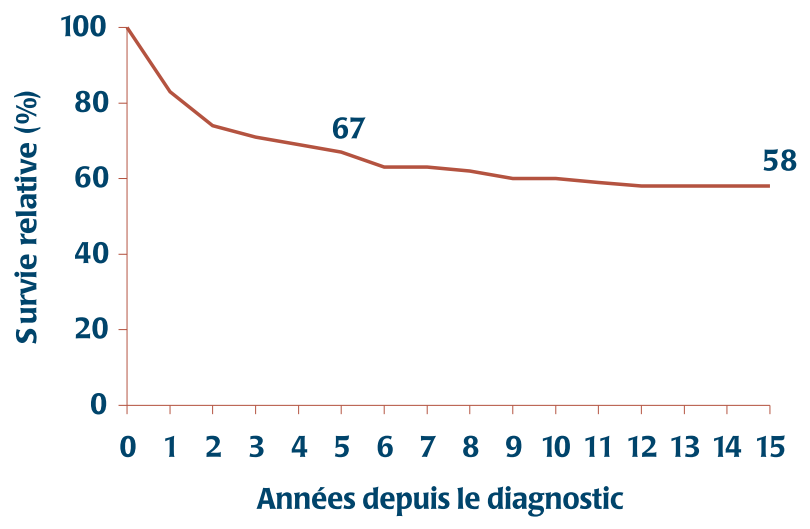


FIGURE 4

Survie relative (\%) à 5 ans par groupe d'âge et selon le siège (tous types de morphologies), Québec, 1984-1995

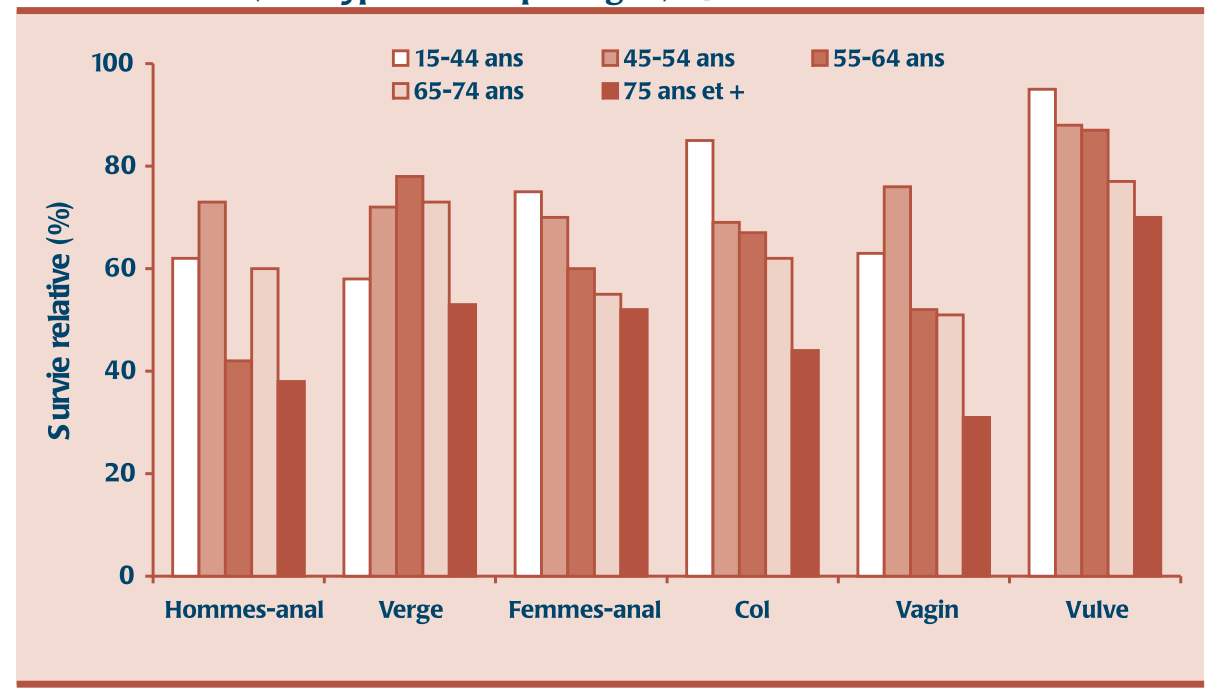

de suivi. Alors que la survie relative a atteint un plateau à 6 ans et à 9 ans après le diagnostic respectivement pour le cancer de la vulve et celui du vagin, elle a continué à diminuer pour le cancer de la verge et pour le cancer anal pour atteindre respectivement $59 \%$ et $42 \% 15$ ans après le diagnostic.

La survie relative varie également selon le groupe d'âge. Pour la plupart des sièges, elle diminue avec l'âge (figure 4). Toutefois, pour le cancer du vagin, le cancer anal chez les hommes et le cancer de la verge, la survie relative a un profil différent pour le groupe d'âge le plus jeune. En effet, pour ce groupe d'âge, elle n'est pas la plus élevée comparativement aux autres sièges ano-génitaux de cancer.

\section{Évolution de l'incidence des cancers ano-génitaux}

L’incidence du cancer anal a augmenté entre 1984-1986 et 1999-2001, particulièrement chez les femmes (figure 2). Le cancer de la vulve semble aussi connaître une légère augmentation à partir de la période 1993-
FIGURE 5

Survie relative (\%) à 5 ans par période du diagnostic et selon le siège (tous types de morphologies), Québec, 1984-1986 à 1993-1995

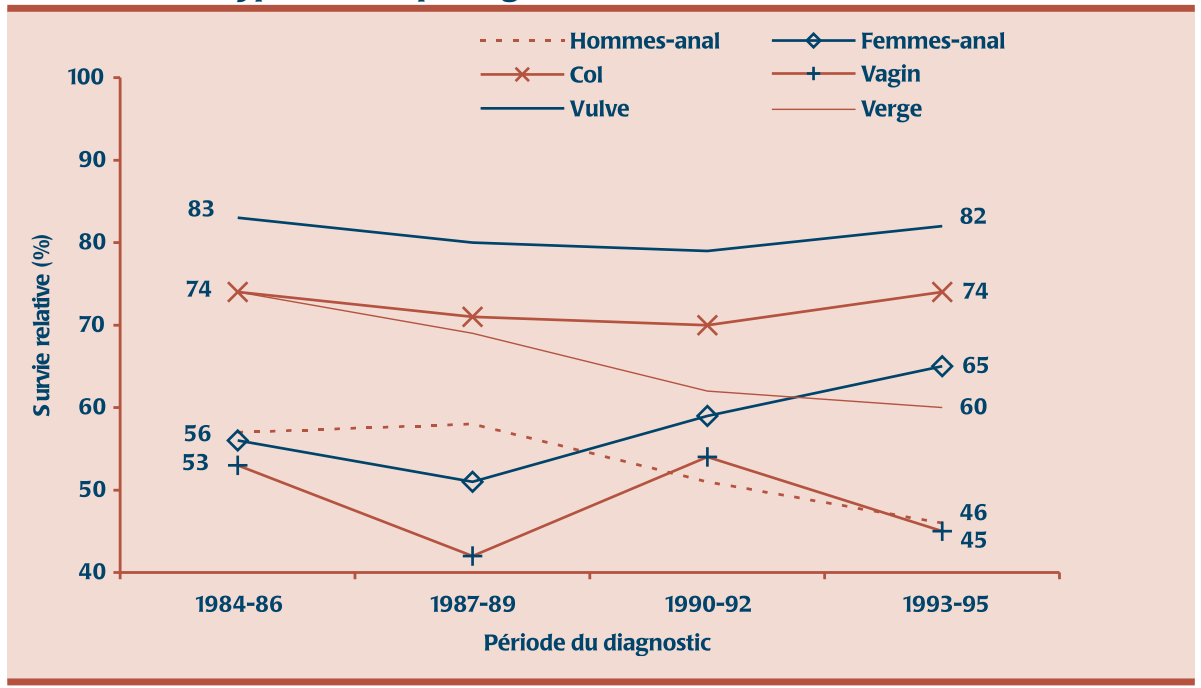

95. Par contre, pour le cancer du col de l'utérus et le cancer du vagin, on remarque une diminution au fil des années.

\section{Évolution de la survie relative des cancers ano-génitaux}

Pour le cancer anal et de la verge, la survie relative a baissé au fil des ans chez les hommes, passant respectivement de $57 \%$ et de $75 \%$ pour la période 1984-86 à $46 \%$ et à $59 \%$ pour la période 1993-95. Par contre, chez les femmes, la survie relative à 5 ans a augmenté pour le cancer anal, passant ainsi de $56 \%$ à $65 \%$. Elle est restée stable autour de $74 \%$ pour le cancer du col utérin et $82 \%$ pour le cancer de la vulve (figure 5).

\section{Association entre l'incidence du cancer anal et le cancer du col chez les femmes au Québec entre 1984 et 1999 : incidence d'un deuxième cancer}

Nous avons également examiné l'association entre le cancer anal et le cancer du col utérin chez les femmes. Seulement 3 femmes parmi les 5818 qui ont eu un cancer du col ou un cancer anal ont aussi eu un deuxième cancer de siège différent (col ou anal) au cours d'une période d'observation de 17 ans au Québec.

\section{Discussion}

Les résultats de cette étude montrent que, de 1984 à 2001, au Québec, l’incidence des cancers ano-génitaux est en augmentation bien qu'elle soit faible, particulièrement pour le cancer anal chez les femmes et pour les cancers de la vulve à partir de la période de 1993-95. La même tendance a été observée d'une part pour le cancer anal, dans plusieurs pays, notamment les ÉtatsUnis, le Danemark et la Suède $\mathrm{e}^{(5,6,8,9,19,20)}$ et d'autre part pour le cancer de la vulve, entre la période de 1958-69 et la période de 1990-96, en Suède ${ }^{(21)}$ et entre la période de 1985-88 et celle de 1994-97, en Autriche ${ }^{(22)}$. Cependant, cette dernière élévation n'a pas été observée en Norvège, en Suisse et aux États-Unis ${ }^{(11,23,24)}$. La hausse des néoplasies intraépithéliales de la vulve (VIN), état précurseur du cancer de la vulve ${ }^{(24)}$, parti- 
culièrement chez les jeunes femmes ${ }^{(25)}$, pourrait expliquer l'augmentation observée de l'incidence de cancer de la vulve. Dans une étude effectuée en Nouvelle-Zélande, on a rapporté que $87,5 \%$ des VIN de grade 3 chez 113 femmes qui n'ont pas été traitées ont évolué vers un cancer invasif $^{(26)}$.

Contrairement aux autres cancers anogénitaux, les taux d'incidence des cancers du col de l'utérus et du vagin ont connu une baisse très marquée. La baisse de l'incidence des cancers du vagin a été aussi observée en Suisse. En effet, le taux d'incidence de cancer du vagin (pour 100000 ) a diminué de 0,8 pour la période 1974-84 à 0,4 pour la période $1985-94^{(11)}$. Par contre, en Suède, c'est une légère augmentation qui a été observée $\mathrm{e}^{(21)}$. Pour les cancers du col, les efforts de dépistage précoce, avec l'introduction du test cytologique de Papanicolaou dans les années ' 60 expliquent la réduction du nombre de cancers du col utérin. Le retrait en 1971 du diethylstibestrol (D.E.S.) pourrait aussi expliquer le déclin de cette incidence et de celle de cancer du vagin. Le D.E.S., une hormone œstrogène de synthèse, a été trouvée associée à un risque accru de néoplasies intraépithéliales de type épidermoïde et d'adénocarcinomes à cellules claires du col utérin et du vagin chez les filles dont les mères ont été traitées pendant la grossesse par ce médicament ${ }^{(27)}$. Cette hormone a été prescrite, entre 1941 et 1971, aux femmes ayant des problèmes de saignement durant leur grossesse, qui avaient déjà fait des fausses couches ou qui souffraient de diabète ${ }^{(28)}$.

L'infection au VPH dans la sphère génitale soulève la question du risque de cancers multiples, particulièrement chezles femmes pour les cancers anaux et du col utérin. $\mathrm{Au}$ Québec, sur une période de 17 ans, seulement trois femmes ont eu un cancer anal et un cancer du col. Ce résultat ne nous permet pas d'établir une association claire entre ces deux sièges de cancer. Par contre, dans une étude rétrospective, mesurant les ratios d'incidence standardisés entre les néoplasies intraépithéliales du col, à bas et haut grade (NIC 1, 2 et 3) et l'incidence des cancers ano-génitaux concomitants et subsuquents (le col, l'anus, la vulve et le vagin), une forte association a été démontrée ${ }^{(29)}$.

Les résultats ont également montré que la survie relative est plus faible chez les plus jeunes pour le cancer anal chez les hommes et pour les cancers de la verge et du vagin. Des résultats contraires pour le cancer anal ont été rapportés dans une étude menée par Jeffreys et coll. 2006, en Angleterre et aux Pays de Galles ${ }^{(30)}$. Aussi, une différence importante a été observée dans notre étude entre les hommes et les femmes quant à la survie relative au cancer anal. L'écart ne cessant d'augmenter a atteint une différence de $19 \%$ pour la période de 1993-95. Alors que la survie relative s'est améliorée chez les femmes, elle a baissé chez les hommes. Le même résultat a été observé aux Etats-Unis ${ }^{(7)}$. Par contre, en Angleterre et aux Pays de Galles, la survie à 5 ans au cancer anal s'est améliorée chez les hommes entre 1986 et $2001^{(30)}$.

Le cancer anal est plus fréquent chez les hommes, qui ont des relations sexuelles anales réceptives et qui sont infectés par $\mathrm{VIH}^{(9)}$. Au Québec, dans une étude menée par Goggin et coll. 2005, on a rapporté que le taux d'incidence du cancer anal de type épidermoïde, est en augmentation de façon importante chez les hommes dans la région de Montréal ${ }^{(31)}$. En fait, entre 1984 et 2001, l'incidence des cancers anaux de type épidermoïde a augmenté de $353 \%$ chez les hommes et $134 \%$ chez les femmes dans la région métropolitaine de Montréal. Par contre, aucune augmentation de l'incidence du cancer anal de type adénocarcinome, qui constitue environ $21 \%$ de cas, n'a été observée durant la même période. L'accès à la thérapie antirétrovirale peut allonger la survie des personnes atteintes du VIH mais présentant une immunodéficience chronique, favorisant le développement de cancer anal et ainsi une augmentation de son incidence. Cependant, ces mêmes personnes risquent d'avoir un mauvais pronostic après leur diagnostic de cancer, ce qui pourrait expliquer en partie la faible survie observée pour ces cancers.

La prévention secondaire par le dépistage précoce des lésions précancéreuses du cancer du col utérin et la prévention primaire par la vaccination offrent la meilleure option pour réduire le fardeau de la maladie car elles permettent de réduire l'incidence. Généralement, à l'étape de cancer, l'efficacité du traitement semble avoir atteinte un plateau et la mortalité ne diminue plus. D’ailleurs, au Québec, la survie relative, qui est un indicateur de l'efficacité des traitements, n'a pas changé au fil des ans pour le cancer du col et pour la plupart des principaux sièges de cancer $^{(18)}$.

En conclusion, au Québec, bien que le nombre de cas incident pour ces cancers soit relativement faible comparé aux autres sièges de cancer, il est en augmentation continue et la survie qui y est associée est médiocre chez les hommes en plus d'être constamment en baisse. Le fardeau relié à l'infection par le VPH reste quand même très important. La venue de vaccins prophylactiques contre le VPH est une mesure de prévention primaire qui devrait permettre une protection contre le VPH en complémentarité à la prévention secondaire permise par le dépistage dans le cas du cancer du col.

\section{Remerciements}

Nous tenons à remercier $M$. Michel Beaupré, du ministère de la Santé et des Services sociaux, M. Bernard Duval, de l'Institut national de santé publique du Québec et M. Alain Demers, de CancerCare Manitoba, pour leurs commentaires judicieux qui ont contribué à finaliser ce document. 


\section{Références}

1. Franco EL, Harper DM. Vaccination against human papillomavirus infection: a new paradigm in cervical cancer control. Vaccine 2005;23:2388-94.

2. Parkin DM, Bray F. Chapter 2: The burden of HPV-related cancers. Vaccine 2006;24 Suppl 3:S11-S25.

3. Spence AR, Franco EL, Ferenczy A. The Role of Human Papillomaviruses in Cancer. Evidence to Date. Am J Cancer 2005; 4:49-64.

4. Gillison ML, Shah KV. Chapter 9: Role of mucosal human papillomavirus in nongenital cancers. J Natl Cancer Inst Monographs 2003;57-65.

5. Melbye M, et coll. Changing patterns of anal cancer incidence in the United States, 19401989. Am J Epidemiol 1994;139:772-80.

6. Maggard MA, Beanes SR, Ko CY. Anal canal cancer: a population-based reappraisal. Dis Colon Rectum 2003;46:1517-23.

7. Johnson LG, et coll. Anal cancer incidence and survival: the surveillance, epidemiology, and end results experience, 1973-2000. Cancer 2004;101:281-8.

8. Goldman S, et coll. Incidence of anal epidermoid carcinoma in Sweden 19701984. Acta chirurgica Scandinavica 1989;155:191-7.

9. Frisch M, Melbye M, Moller H. Trends in incidence of anal cancer in Denmark. BMJ (Clinical research ed.) 1993;306:419-22.

10. Cheon J, et coll. Survey of incidence of urological cancer in South Korea: a 15-year summary. Int J Urol 2002;9:445-54.

11. Levi F, Randimbison L, La Vecchia C. Descriptive epidemiology of vulvar and vaginal cancers in Vaud, Switzerland, 1974-1994. Ann Oncol 1998;9:1229-32.
12. Maiche AG. Epidemiological aspects of cancer of the penis in Finland. Eur J Cancer Prev 1992;1:153-8.

13. Louchini R. La survie au cancer pour les nouveaux cas déclarés au Québec en 1992 qualité des données, probabilité de survie relative à cinq ans. Montréal : Institut national de santé publique, Direction du développement et des programmes, Unité connaissance-surveillance, 2002.

14. Duchesne D, et coll. Life tables, Canada, provinces and territoies, 1995-1997. Ottawa : Statistique Canada, 2002.

15. Millar WJ, David P, Statistique Canada. Life tables, Canada and provinces, 19901992. Ottawa : Statistique Canada, 1995.

16. Statistics C. Life tables, Canada and provinces, 1986. Ottawa : Statistique Canada, 1990.

17. Ederer $F$, Heise $H$. Instructions to IBM 650 programmers in processing survival computations. Methodological note No.10, End Results Evaluation Section. 1959. National Cancer Institute, Bethesda MD.

18. Louchini R, Beaupré M. La survie reliée au cancer pour les nouveaux cas déclarés au Québec, de 1984 à 1998 : survie observée et survie relative. 2003.

19. Cress RD, Holly EA. Incidence of anal cancer in California: increased incidence among men in San Francisco, 1973-1999. Prev Med 2003;36:555-60.

20. Diamond C, et coll. Increased incidence of squamous cell anal cancer among men with AIDS in the era of highly active antiretroviral therapy. Sex Transm Dis 2005;32:314-20.

21. Hemminki $\mathrm{K}$, Li $\mathrm{X}$, Vaittinen P. Time trends in the incidence of cervical and other genital squamous cell carcinomas and adenocarcinomas in Sweden, 19581996. Eur J Obstet Gynecol Reprod Biol 2002;101:64-9.
22. Joura EA, et coll. Trends in vulvar neoplasia. Increasing incidence of vulvar intraepithelial neoplasia and squamous cell carcinoma of the vulva in young women. $\mathrm{J}$ Reprod Med 2000;45:613-5.

23. Sturgeon SR, et coll. In situ and invasive vulvar cancer incidence trends (1973 to 1987). Am J Obstet Gynecol 1992;166:1482-5.

24. Iversen $\mathrm{T}$, Tretli S. Intraepithelial and invasive squamous cell neoplasia of the vulva: trends in incidence, recurrence, and survival rate in Norway. Obstet Gynecol 1998;91:969-72.

25. Jones RW, Baranyai J, Stables S. Trends in squamous cell carcinoma of the vulva: the influence of vulvar intraepithelial neoplasia. Obstet Gynecol 1997;90:448-52.

26. Jones RW, Rowan DM. Vulvarintraepithelial neoplasia III: a clinical study of the outcome in 113 cases with relation to the later development of invasive vulvar carcinoma. Obstet Gynecol 1994;84:741-5.

27. Hatch EE, et coll. Incidence of squamous neoplasia of the cervix and vagina in women exposed prenatally to diethylstilbestrol (United States). Cancer Causes Control 2001;12:837-45.

28. D.E.S. Action Canada. Le D.E.S. http:// www.web.net/ desact/francais/francais. html . 2007.

29. Kalliala I, et coll. Risk of cervical and other cancers after treatment of cervical intraepithelial neoplasia: retrospective cohort study. BMJ (Clinical research ed.) 2005;331:1183-5.

30. Jeffreys M, et coll. Survival from rectal and anal cancers in England and Wales, 19862001. Eur J Cancer 2006;42:1434-40.

31. Goggin P, Louchini R, Steben M. Trends in anal cancer incidence in Quebec. 2005. 22nd International Papillomavirus Conference and Clinical Worshop 2005. Abstract no. P-037. 